\title{
Recognition and treatment of atrial fibrillation caused by accessory pathway in a middle-aged patient
}

\author{
Abdelrahman M. Aljadi MD ${ }^{1}$, Esam Baryun $\mathrm{MD}^{1}$
}

\section{Author Affiliations:}

1. Marshall University Joan C. Edwards School of Medicine, Huntington, West Virginia

The authors have no financial disclosures to declare and no conflicts of interest to report.

\section{Corresponding Author:}

\author{
Abdelrahman M. Aljadi MD \\ Marshall University \\ Huntington, West Virginia \\ Email: aljadi@marshall.edu
}




\section{Abstract}

Atrial fibrillation (AF) management is an everyday healthcare practice at the emergency department, inpatient setting, and outpatient setting. It is a priority to identify and treat reversible causes of AF before committing the patient to an unnecessary lifelong medical regimen that may include antiarrhythmics and anticoagulants. We report a case of a middle-aged patient with recurrent episodes of AF triggered by a concealed Accessory Pathway (AP). He was on Rivaroxaban and Sotalol for years, then later successfully treated with catheter ablation of the accessory pathway. A 48-year-old white Caucasian male with a history of recurrent episodes of AF was initially treated with Rivaroxaban and Sotalol, and underwent electrical cardioversions four times. He eventually had a successful catheter ablation after a comprehensive electrophysiological study had revealed a concealed AP. He is currently off Rivaroxaban and Sotalol. This case highlights the importance of detection and treatment of the reversible causes of atrial fibrillation in young and middle-aged patients. By applying this practice, physicians will be able to avoid the unnecessary use of anticoagulation and anti-arrhythmic therapy in young and middle-aged patient groups.

\section{Keywords}

Atrial fibrillation (AF), Accessory Pathway (AP), radiofrequency ablation, anticoagulation therapy, antiarrhythmic therapy

\section{Introduction}

Atrial fibrillation (AF) is the most common cardiac arrhythmia in clinical practice. The prevalence and the incidence of AF increases with advanced age. ${ }^{1}$ Epidemiological data estimates that the number of individuals with AF in 2010 was 33.5 million. ${ }^{2,3}$ Hypertensive heart disease and coronary artery disease remain the most common chronic underlying cardiac cases of AF. ${ }^{4}$ Less common cardiac causes of AF include valvular heart disease, heart failure, hypertrophic cardiomyopathy, congenital heart disease, and pericarditis. Pulmonary risk factors of AF include COPD and pulmonary embolism. Increased pulmonary vascular resistance due to chronic venous thromboembolic disease has also contributed to the development of AF. Other miscellaneous causes of AF include a BMI of greater than $30^{5}$, diabetes mellitus, metabolic syndrome, and chronic kidney disease.

Potentially reversible risk factors of AF in young patients include acute alcohol intoxication (holiday heart), thyrotoxicosis, cardiothoracic surgery and non-cardiothoracic surgery. Autonomic dysfunction with increased sympathetic tone may play a role in the development of $\mathrm{AF}$ especially in the paroxysmal type. Evidence from previous studies by Sharma AD, et $\mathrm{al}^{6}$ indicate that the accessory pathway may play a major role in the development of atrial fibrillation. It also has been reported in an old study by Wellen, et al that patients with a concealed accessory pathway as well as patients with Wolff-Parkinson-White syndrome could be expected to have an incidence of atrial fibrillation. ${ }^{7,8}$ Explanations for the occurrence of AF in these patients are not fully clear, but a proposed mechanism is that a retrograde conduction of the ventricular beat degenerates into AF in the atrium. ${ }^{9}$ Further studies are needed to uncover the exact mechanism of atrial fibrillation in patients with concealed accessory pathway. 


\section{Case presentation}

We report a case of a 48-year-old Caucasian male with a history of CAD and multiple stents, anterior wall MI, AF status post electrical cardioversions, on Rivaroxaban and Sotalol therapy. He presented with recurrent tachypalpitations associated with dizziness and light-headedness; no chest pain or syncope. His social history was negative for any alcohol intake. Initial EKG showed narrow complex regular tachycardia, 172 beats per minute, no Delta wave, no clear P waves, but possible $\mathrm{P}$ wave on ST segment in V1 \& II. The physical exam showed stable blood pressure and respiratory rate. Cardiac exam revealed regular tachycardia, without murmurs, gallops or rubs. TSH was normal and Troponin was negative. He received Digoxin intravenously in the ER; tachycardia persisted but QRS became wide. Amiodarone was given IV for possible ventricular tachycardia, and he converted to sinus rhythm.

Since our patient was young and presented with a regular tachycardia, we suspected the presence of reentry supraventricular tachycardia (SVT) as a cause of AF. It has been reported that SVT in general can cause AF even without accessory pathway. ${ }^{10}$ An electrophysiology study was done and revealed a left concealed accessory pathway, inducible Orthodromic Reentry Tachycardia (ORT), and no pre-excitation. Radio-frequency ablation of the AP was successful and ORT was not inducible. It was determined that the patient may not need anticoagulants and antiarhythmic agents. We felt comfortable stopping Sotalol and Rivaroxaban. He was seen at his follow up appointment and was doing well. Heart monitors showed normal rhythm with no AF. The patient was happy to be off medications he had taken for years, and thought he was going to be taking forever.



EKG1. Sinus Tachycardia

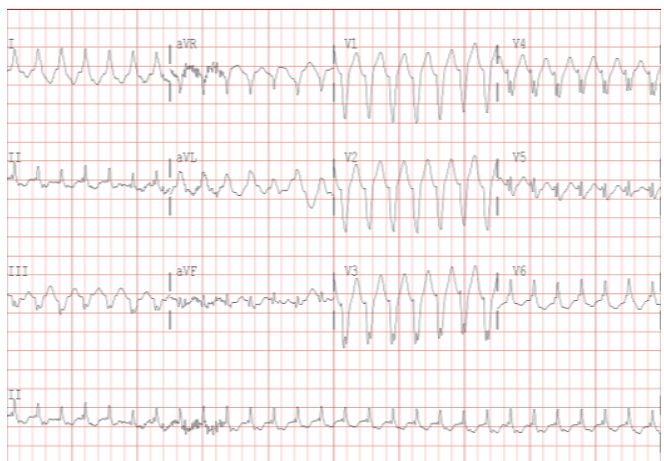

EKG2. Wide complex tachycardia

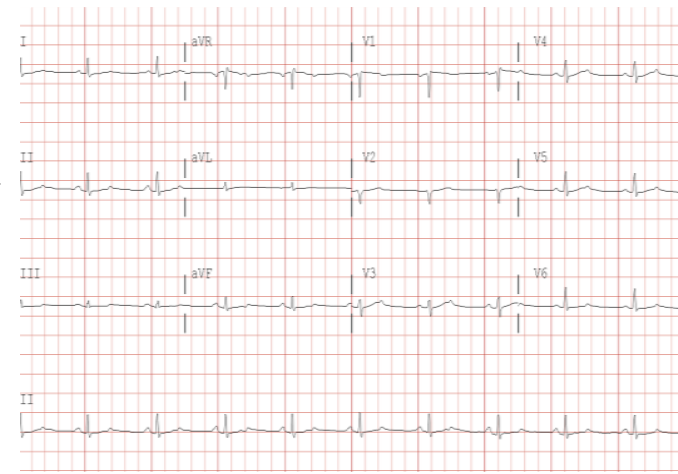

EKG 3. Sinus rhythm. 

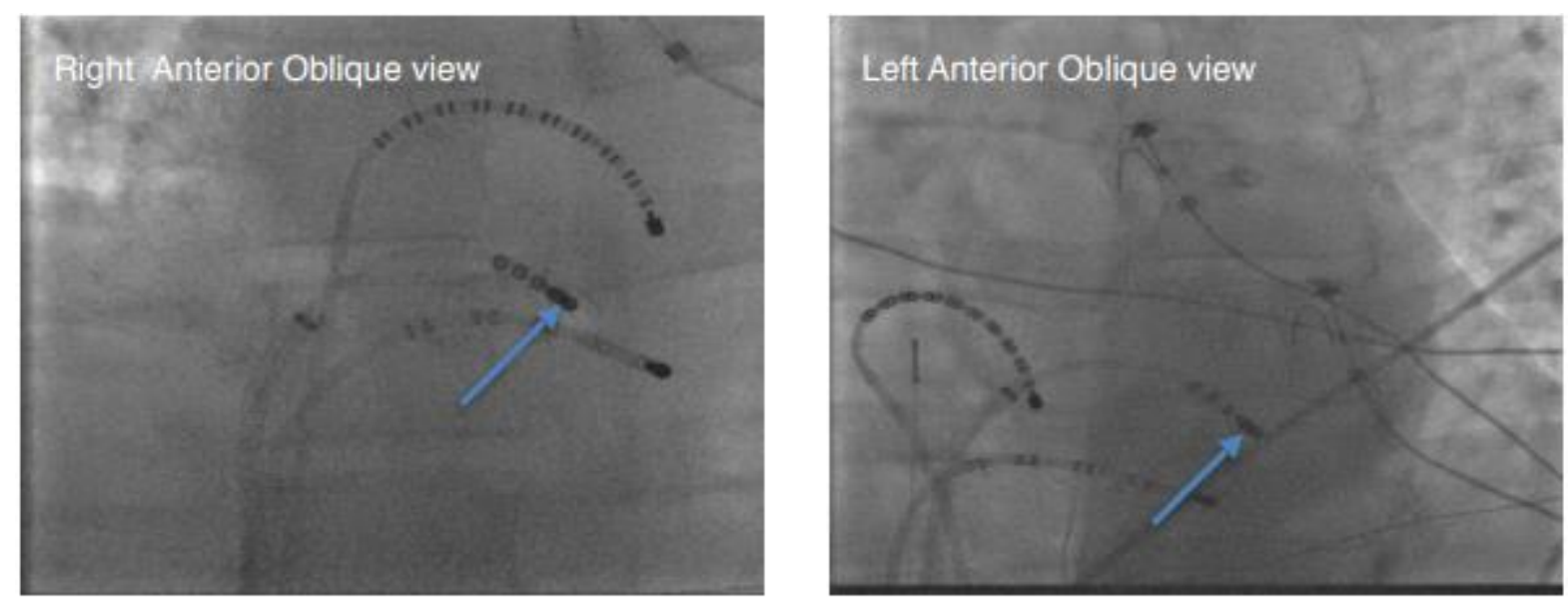

Figure 1-2: Arrows show tip of ablation catheter placed over accessory pathway in left atrium via transeptal sheath

\section{Discussion}

AF in young patients can be trigged by multiple reversible causes such as acute alcohol intoxication, thyrotoxicosis, sleep apnea, and accessory pathway. It is a priority to rule out reversible causes. Regular tachycardia on presentation raises suspicion for reentry SVT, focal atrial tachycardia, and atrial flutter (AFL). AFL can occur in AF patients who take antiarrhythmics, and is usually treated with a flutter ablation plus continuation of antiarrhythmic medications. Reentry SVT can cause AF, hence management includes SVT ablation and discontinuation of anti-arrhythmic agents. Adenosine is a good option to help reveal flutter waves. It may reveal atrial tachycardia if it doesn't terminate it (adenosine terminates 20\% of focal atrial tachycardia).

The P wave in typical atrioventricular nodal tachycardia (AVNRT) occurs within $70 \mathrm{~ms}$ of QRS initiation, hence it is masked by QRS or falls immediately after and appears as a pseudo R in V1 or pseudo $\mathrm{S}$ in inferior leads. The $\mathrm{P}$ wave in atypical AVNRT occurs before the QRS in a long RP pattern. $\mathrm{P}$ waves in ORT usually fall on the ST segment. The notch suspected to be a P wave in this case fell on ST segment beyond $80 \mathrm{~ms}$ of QRS initiation, therefore more likely ORT. The widening of QRS after digoxin and history of old MI added VT to the differential diagnosis. Due to the fact that the initial QRS upstroke in lead II and V5 in the second EKG was similar to the native QRS, and the delay was at the end of QRS complex, we interpreted these EKG changes as aberrancy, not pre-excitation.

Accessory pathways can conduct antegrade \& retrograde, or retrograde only (concealed). EPS showed a left concealed accessory pathway. ORT was easily induced and sustained. We got access into the left atrium via trans septal approach (from femoral vein to RA into LA). Retrograde access via femoral artery across aortic valve into LV then MV annulus is an option. ORT was not induced after ablation.

Radiofrequency catheter ablation was a successful treatment to prevent atrial fibrillation recurrence and to get the patient off Rivaroxaban and Sotalol. 


\section{Conclusion}

We would like to emphasize the importance of recognition of concealed accessory pathway as a cause of reversible atrial fibrillation in young and middle aged patients. We can save these patients from being on lifelong anticoagulation and ant-arrhythmic treatment by managing them with radiofrequency ablation treatment. 


\section{References}

1. Chugh SS, Blackshear JL, Shen WK, Hammill SC, Gersh BJ. Epidemiology and natural history of atrial fibrillation: clinical implications. J Am Coll Cardiol. Feb 2001;37(2):371-378.

2. Lip GY, Brechin CM, Lane DA. The global burden of atrial fibrillation and stroke: a systematic review of the epidemiology of atrial fibrillation in regions outside North America and Europe. Chest. Dec 2012;142(6):1489-1498.

3. Chugh SS, Havmoeller R, Narayanan K, et al. Worldwide epidemiology of atrial fibrillation: a Global Burden of Disease 2010 Study. Circulation. Feb 25 2014;129(8):837-847.

4. Kannel WB, Abbott RD, Savage DD, McNamara PM. Epidemiologic features of chronic atrial fibrillation: the Framingham study. N Engl J Med. Apr 29 1982;306(17):1018-1022.

5. Nalliah CJ, Sanders P, Kottkamp H, Kalman JM. The role of obesity in atrial fibrillation. Eur Heart J. May 21 2016;37(20):1565-1572.

6. Sharma AD, Klein GJ, Guiraudon GM, Milstein S. Atrial fibrillation in patients with Wolff-ParkinsonWhite syndrome: incidence after surgical ablation of the accessory pathway. Circulation. Jul 1985;72(1):161-169.

7. Wellens HJ, Durrer D. The role of an accessory atrioventricular pathway in reciprocal tachycardia. Observations in patients with and without the Wolff-Parkinson-White syndrome. Circulation. Jul 1975;52(1):58-72.

8. Farshidi A, Josephson ME, Horowitz LN. Electrophysiologic characteristics of concealed bypass tracts: clinical and electrocardiographic correlates. Am J Cardiol. May 22 1978;41(6):1052-1060.

9. Campbell RW, Smith RA, Gallagher JJ, Pritchett EL, Wallace AG. Atrial fibrillation in the preexcitation syndrome. Am J Cardiol. Oct 1977;40(4):514-520.

10. Hamer ME, Wilkinson WE, Clair WK, Page RL, McCarthy EA, Pritchett EL. Incidence of symptomatic atrial fibrillation in patients with paroxysmal supraventricular tachycardia. J Am Coll Cardiol. Apr 1995;25(5):984-988. 